\title{
Achieving zero stress in iridium, chromium, and nickel thin films
}

\author{
${ }^{a}$ David M. Broadway, ${ }^{b}$ Jeffrey Weimer, ${ }^{b}$ Danielle Gurgew, ${ }^{b}$ Tomasz Lis, ${ }^{a}$ Brian D. Ramsey, \\ ${ }^{a}$ Stephen L. O’Dell, ${ }^{c}$ A. Ames, and ${ }^{c} R$. Bruni \\ ${ }^{a}$ NASA Marshall Space Flight Center, Huntsville, AL 35812, USA \\ bUniversity of Alabama in Huntsville, Huntsville, AL 35899, USA \\ ${ }^{\mathrm{c}}$ Smithsonian Astrophysical Observatory, Cambridge, MA 02140, USA
}

\begin{abstract}
We examine a method for achieving zero intrinsic stress in thin films of iridium, chromium, and nickel deposited by magnetron sputter deposition. The examination of the stress in these materials is motivated by efforts to advance the optical performance of light-weight $\mathrm{x}$-ray space telescopes into the regime of sub-arc second resolution that rely on control of the film stress to values within 10-100 MPa. A characteristic feature of the intrinsic stress behavior in chromium and nickel is their sensitivity to the magnitude and sign of the intrinsic stress with argon gas pressure, including the existence of a critical pressure that results in zero film stress. This critical pressure scales linearly with the film's density. While the effect of stress reversal with argon pressure has been previously reported by Hoffman and others for nickel and chromium, we have discovered a similar behavior for iridium. Additionally, we have identified zero stress in iridium shortly after island coalescence. This feature of film growth is used for achieving a total internal stress of -2.89 MPa for a $15.8 \mathrm{~nm}$ thick iridium film. The surface roughness of this low-stress film was examined using scanning probe microscopy (SPM) and $\mathrm{x}$-ray reflectivity (XRR) at $\mathrm{CuK} \alpha$ and these results presented and discussed.
\end{abstract}

Key words: $x$-ray space telescopes, soft $x$-ray optical coatings, in-situ measurement of film stress, thin film characterization, zero stress iridium thin films 


\section{INTRODUCTION}

The control of film stress is a leading technological challenge in the development of the next generation of high resolution lightweight $\mathrm{x}$-ray space telescopes. The strict weight constraints combined with the concentric nesting of a large number of optics, which is needed to maximize the number of x-ray photons captured from faint celestial sources, can result in substrate thicknesses of only a few tens of microns.

Materials which might be attractive for their physical characteristics can otherwise be disqualified due to large internal stress. Choices for high density metals for x-ray reflectivity, for example, often include gold (Au), platinum (Pt), and iridium (Ir). Among these metals, iridium has the highest density and reflectivity in the soft X-ray spectra and is often a preferred material for these reasons. Despite iridium's superior x-ray optical performance it renders high compressive stress on the order of a few gigapascal. In order to reduce the stress in the compressive iridium layer, others have attempted to first deposit a chromium layer with tensile stress. The thickness of the chromium layer is increased until a balance is reached in the net stress of the $\mathrm{Cr} / \mathrm{Ir}$ bi-layer ${ }^{1,2,3}$. This method has resulted in a successful reduction in the net stress, but the tensile stress in underlying chromium layer is associated with columnar film growth and results in an increase in surface roughness that is expected to worsen with increasing film thickness.

Methods of stress reduction are being evaluated for an adaptive x-ray optic application that is being led by The Smithsonian Astrophysical Observatory (SAO) ${ }^{4,5}$. Here a thin-film piezoelectric (PZT, lead zirconate titanate) ceramic actuator array with high tensile stress (i.e. $150 \mathrm{~N} / \mathrm{m})$ is deposited on one surface of a thin $(\sim 0.4 \mathrm{~mm})$ slumped glass segment, while the opposing surface is coated with an $\mathrm{x}$-ray reflective layer, such as iridium. If the $\mathrm{Cr} / \mathrm{Ir}$ bi-layer approach is used, however, the chromium layer must not only compensate the stress in the iridium layer, but also the high tensile stress in the piezo layer. This approach would result in a thick compensation layer and high surface roughness at the Ir air interface. Therefore, any reduction in the compressive stress of the iridium layer by an alternative method would be beneficial since this would reduce, or possibly eliminate, the thickness of the chromium under-layer.

The behavior of the stress in polycrystalline metals deposited by DC magnetron sputtering has been studied and reported extensively by Hoffman and others ${ }^{6,7,8,9}$. A characteristic feature of the intrinsic stress behavior in materials such as nickel and chromium is the impact to the sign and magnitude of the film stress with argon process pressure including the existence of a critical pressure that results in zero intrinsic stress. We have recently discovered the ability to achieve zero stress in iridium films through the optimization of the process pressure as well.

In the following sections we will examine the in-situ stress in single layer films of nickel, chromium, and iridium deposited on silicon substrates using an instrument recently developed at NASA MSFC. The evaluation of the surface roughness, adhesion, and film density for iridium films in the various states of stress is also examined through XRR (8 $\mathrm{keV}$ ) measurement and analysis of the films which were conducted at SAO. A similar evaluation of the film quality for nickel and chromium will eventually be carried out as well.

\section{IN-SITU STRESS MEASUREMENT INSTRUMENT}

NASA's development of an in-situ instrument measurement technique is driven by efforts to advance the performance of $\mathrm{x}$-ray astronomical telescopes through improved angular resolution, increased aperture area and higher working photon energies. A detailed description of the device can be found elsewhere ${ }^{10}$. However, in light of the leading technological challenge film stress presents in the fabrication of light-weight x-ray space telescopes, a brief overview of its methodology and sensitivity is thought to be appreciated and will be reviewed here as well.

Since the inception and testing of the prototype device, improvements to the instrument in the embodiment presented herein have been realized and are ongoing including: the capability to measure film stress on transparent substrates, 
substrate heating, and greater flexibility in its orientation. Meanwhile, we have used it in its currently described form to effectively study the stress in various metal films — including nickel (Ni), chromium (Cr), and iridium (Ir).

\subsection{In-situ methodology}

The stress in the film results in a bending moment of the wafer causing a change of its curvature. From the measurement of this curvature, the plane stress can be calculated using the well-known Stoney equation,

$$
\sigma h_{f}=\frac{E_{s} h_{s}^{2} \kappa}{6\left(1-\vartheta_{s}\right)}
$$

which relates the stress force per unit width, $\sigma h_{f}$, to the substrate curvature, $\kappa$, through a proportionality constant described by the known geometric and mechanical properties of the substratenamely, the substrate's thickness, $h_{s}$, and biaxial modulus, $\frac{E_{S}}{\left(1-\vartheta_{S}\right)}$. The calculation of the in-situ film stress using the Stoney formalism relies on measurement of the relative change in substrate curvature during deposition or thermal annealing. Note that the Stoney equation predicts a constant value of curvature, which implies spherical substrate deformation, due to uniform stress in the film provided the assumptions used in its derivation are met.

For the case of a uniform, isotropic film, the deformation mode of a circular substrate is given by the constant:

$$
A=\sigma h_{f} \frac{D_{s}^{2}}{h_{s}^{3}}
$$

where $D_{s}$ is the diameter of the substrate, and $\sigma$ is the average film stress in a film of thickness, $h_{f}$. In particular, the substrate deformation will agree to within $10 \%$ of the Stoney equation provided the condition $\mathrm{A} \leq 0.2 \mathrm{~A}_{\mathrm{c}}$ is satisfied (for a silicon substrates $\left.A_{c}=680 \mathrm{GPa}\right)^{11}$. If measurement of even larger values of film stress is sought, then the condition, $\mathrm{A}$ $\leq 0.2 \mathrm{~A}_{\mathrm{c}}$ can be satisfied by utilizing a substrate of increased thickness.

To illustrate, we deposited a nickel film on a substrate that satisfies the conditions of Eqn. (2), and confirm the resulting spherical deformation of the substrate through ex-situ measurement of the film stress. Repeated measurements of the substrate's displacement profile along two orthogonal lines passing through the substrate's center were performed before (pre-trace) and after deposition (post-trace). The curvature can be approximated from the second derivative of this difference for small out-of-plane displacements. Thus, a constant curvature results from a parabolic displacement as a function of scan position. The substrate displacement profiles were measured using a Form Talysurf PGI 1230 for a uniform nickel film deposited on crystalline silicon (111) oriented substrate as shown in Fig. (1). The similarity of the difference traces for two orthogonal scan directions is emphasized in bold. A quadratic fit to the data and the second derivative was evaluated to determine the curvature. The quality of the fits is indicated by the values of the coefficients of determination which is better than three 9's. The evaluation of the second derivative in each direction were in agreement to within $1 \%\left(\kappa_{\mathrm{x}}=0.0212\right.$ and $\left.\kappa_{\mathrm{y}}=0.0214 \mathrm{~m}^{-1}\right)$, indicating nearly spherical deformation of the substrate. Evaluation of the force per unit width resulted in a deformation constant of $\sim 8 \mathrm{GPa}$ which indeed satisfies the constraint for spherical deformation.

Since the stress induced deformation of the substrate is spherical, the curvature is constant over its surface, requiring the measurement of the displacement of only a single point to determine its value. While any point within the interior of the circular substrate may be measured to infer the substrate's constant value of curvature, the displacement at the center yields the highest sensitivity to changes in film stress. The device is therefore akin to the classic spherometer used for measuring the curvature of spherical lens. Our version of this device replaces the central leg with a non-contact vacuum 
compatible optical displacement sensor capable of measuring displacements as small as a few nanometers. Since silicon substrates are not flat, it is the difference in displacement before and during deposition that renders an effective spherical deformation. Therefore, the instrument is zeroed prior to deposition so that this effective curvature is measured.
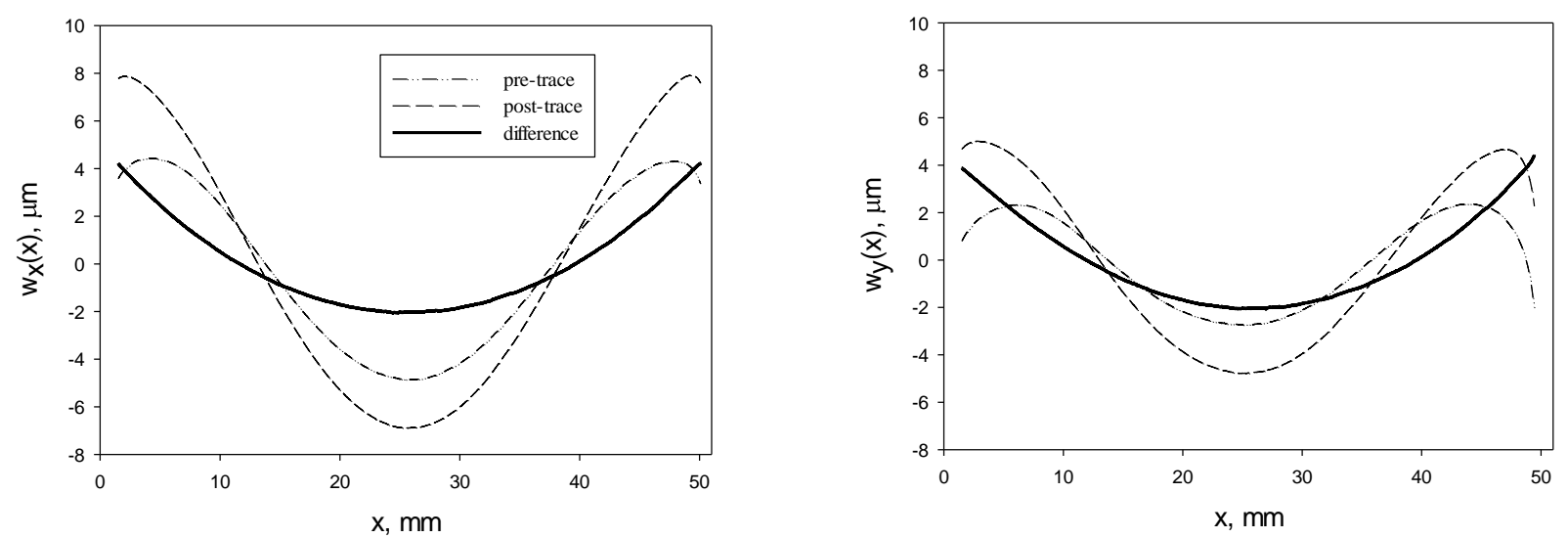

Figure 1. The difference in the pre and post-deposition profilometry traces (effective deformation) for two orthogonal scan directions show a similar parabolic dependence of the stress induced displacement, indicating spherical deformation of the Si (111) substrate. Evaluation of the second derivative of the difference traces result in agreement between two values of curvature to within $1 \%$ (i.e. $\kappa_{\mathrm{x}}=0.0212$ and $\left.\kappa_{\mathrm{y}}=0.0214 \mathrm{~m}^{-1}\right)$.

Figure 2 (a) and (b) illustrates schematically the elements and defining geometry of the spherometer. In order to protect the fiber optic tip from becoming coated during deposition the sensor is located to measure the displacement of the opposing coated surface of a double-side polished substrate.

The spherical curvature is calculated by the direct measurement of the substrate sagitta, $\delta$, for a spherometer constant $r$, (i.e. radial distance of the support pins) through the geometric relation:

$$
\kappa=\frac{2 \delta}{r^{2}+\delta^{2}}
$$

For small displacements, $\delta \ll r$ the Stoney equation can be re-written in terms of $\delta$ as,

$$
\sigma h_{f}=-\frac{1}{3} \frac{E_{s}}{(1-\vartheta s)}\left(\frac{h_{s}}{r}\right)^{2} \delta
$$

The components that constitute the spherometer consist of a substrate holder, three substrate support pins (or legs) with hemispherical tips, and the fiber optic displacement sensor. A thermocouple located in one of the support pins measures a reference temperature that is related to the temperature of the substrate. The displacement sensor has a resolution of 5 $\mathrm{nm}$. It is vacuum compatible to $10^{-7}$ Torr and can tolerate temperatures to $480^{\circ} \mathrm{C}$. 

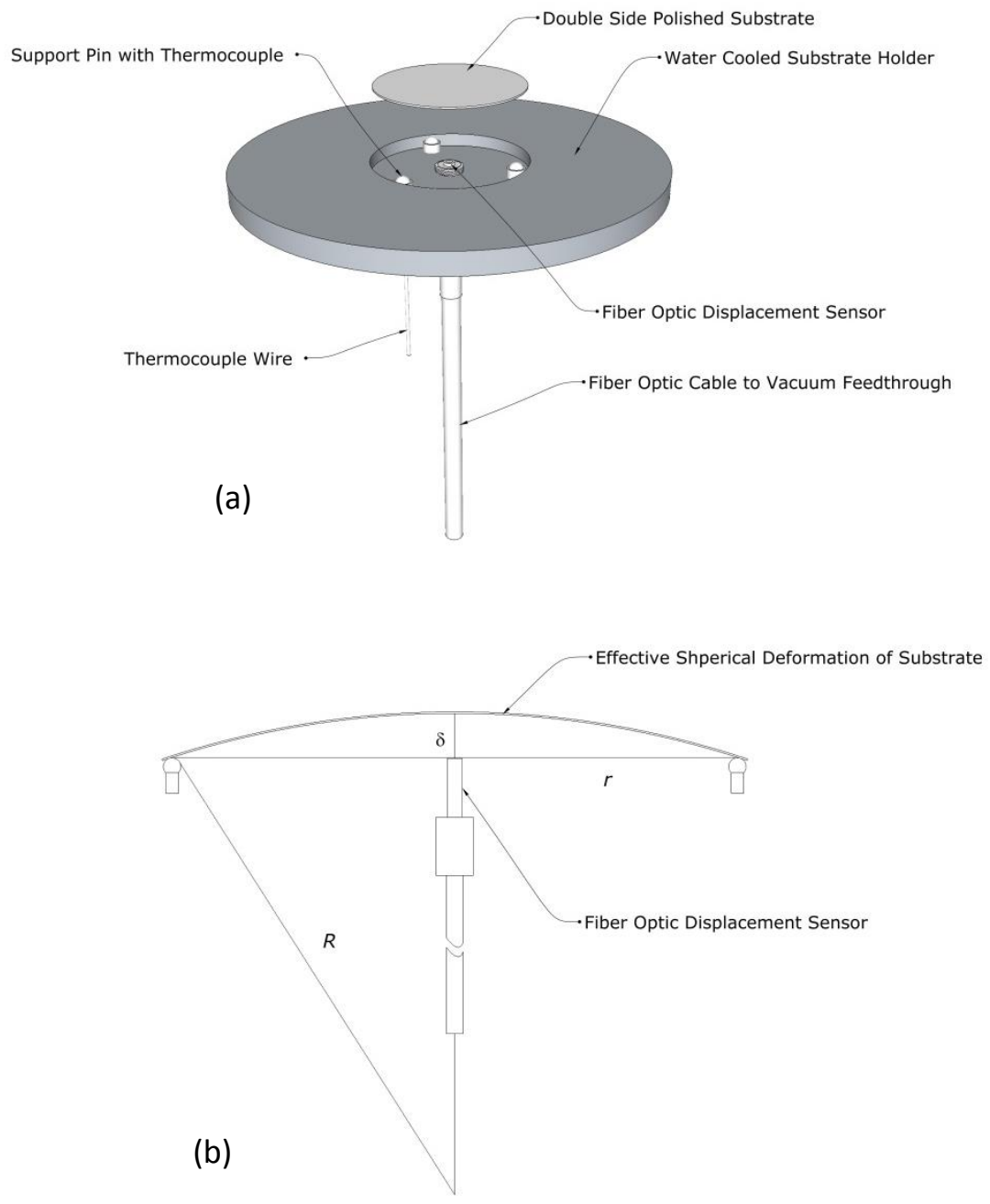

Figure 2. A schematic diagram illustrating the primary components of the non-contact spherometer (a) and defining geometry (b) used in the calculation of the substrate curvature. The relative displacement at the center of the opposing coated surface of a doubleside polished substrate is measured to infer the stress-induced curvature of the spherically deformed substrate.

\subsection{Sensitivity}

We define the sensitivity as the smallest detectable change, or differential, of the force per unit width, $\Delta\left(\sigma \mathrm{h}_{\mathrm{f}}\right)$. This differential force per unit width is related to the minimum detectable displacement, $\Delta \delta$, and depends on the thickness and elastic properties of the substrate according to Eqn. (4).

For most in-situ stress measurement techniques, the sensitivity is limited by the vibrational background that is common in most high vacuum deposition systems. To establish an estimate of the minimum detectable force per unit width of our apparatus, we sampled displacement data at $2.5 \mathrm{~Hz}$ during a thirty-eight minute deposition process for a thermally equilibrated substrate. No vibrational damping mechanisms were used during the measurement. A polynomial regression of the measured displacement data was performed. The residuals from this analysis are thought to relate to the highfrequency random noise which limits the resolution of the displacement sensor, and ultimately limits the minimum 
detectable force per unit width of the instrument. A value of $1.69 \mathrm{~nm}$ for the full width at half maximum (FWHM) was found from the apparent normal distribution of the residuals. Setting $\Delta \delta$ equal to the FWHM and substituting into Eqn. (4) gives an estimate of the sensitivity of our instrument to be $0.015 \mathrm{~N} / \mathrm{m}$ for a $110 \mu \mathrm{m}$ thick Si (111) thermal equilibrated substrate.

In comparison, the minimum detectable curvature for the multiple beam optical stress sensor (MOSS) technique has been reported as $\Delta \kappa=5.94 \times 10^{-4} \mathrm{~m}^{-1}$, which results in a minimum detectable force per unit width of $0.076 \mathrm{~N} / \mathrm{m}$ for a $100 \mu \mathrm{m}$ substrate ${ }^{11}$. It was later reported that the deposition of a $1 \mathrm{~nm}$ thick film onto a $100 \mu \mathrm{m}$ substrate with a stress of $50 \mathrm{MPa}$ can be routinely detected using the MOSS approach ${ }^{12}$, which is an improvement from the previously reported minimum detectable force per unit width to $0.050 \mathrm{~N} / \mathrm{m}$. Others such as Abermann ${ }^{13}$ and Klokholm ${ }^{14,15}$ have reported sensitivities of $0.020 \mathrm{~N} / \mathrm{m}$ used for the in-situ measurement of the stress in metal films using a cantilever micro-balance technique.

\section{EXPERIMENTAL PROCEDURE}

A circular $7.62 \mathrm{~cm}$ diameter DC magnetron AJA International sputtering source was used to deposit the films on crystalline silicon, (111) oriented, 280-300 $\mu \mathrm{m}$ thick, $2.54 \mathrm{~cm}$ diameter substrate. A native oxide layer is presumed to exist on the substrate surface. The substrates were cleaned with isopropyl alcohol prior to deposition. The targets of various material compositions was cooled indirectly through thermal contact with the water cooled magnetron assembly. The target to substrate distance was $7.5 \mathrm{~cm}$ - both the target and grounded substrate were stationary. The circular substrate and cathode are concentric, resulting in an azimuthally symmetric distribution of film thickness and isotropic, plane stress of the film. The 3:1 ratio of target to substrate diameter resulted in a non-uniformity of film thickness to within $\pm 1 \%$. The deposition rate, $\xi$, was measured in-situ using an Inficon Q-Pod quartz crystal monitor. The monitor was calibrated through independent measurement of the films using XRR. The pressure was measured using a MKS capacitance manometer with a \pm 0.01 mTorr resolution. The out of plane displacement of the uncoated side of the wafer's center was measured during deposition using a fiber optic displacement sensor which has a maximum working range of $40 \mu \mathrm{m}$ and a resolution of $5 \mathrm{~nm}$. The data acquisition rate was $2.5 \mathrm{~Hz}$.

\section{RESULTS}

\subsection{Instantaneous or incremental intrinsic stress}

The incremental or instantaneous stress is the rate of change of the force per unit width with film thickness. For the case in which the stress is constant, with no stress relaxation, the time rate of change of the substrate sagittal, $\delta$, can be expressed as:

$$
\frac{d\left(\sigma h_{f}\right)}{d h_{f}}=-\frac{E_{s}}{3 \xi(1-\vartheta s)}\left(\frac{h_{s}}{r}\right)^{2} \frac{d \delta}{d t}
$$

This quantity represents the change in the cumulative stress due to the stress in a film of differential thickness, $\mathrm{dh}_{\mathrm{f}}$, being deposited on the surface of the growing film at a deposition rate $\xi(\mathrm{nm} / \mathrm{s})$. Under steady state process conditions, the dependence of the force per unit width versus film thickness becomes linear shortly after the start of deposition (i.e. $>10$ $\mathrm{nm}$ ) and continues in this fashion for several hundred nanometers. The linear dependence in the displacement represents a regime of film growth where the incremental intrinsic stress is a constant. This behavior has been reported in the literature for magnetron sputtering of several metals including chromium and nickel. We observe the same newly discovered dependence in the displacement for Ir as well. The instantaneous stress is therefore a convenient quantity since for these metals it is a value independent of film thickness. Therefore, in examining the dependence of the instantaneous stress versus argon pressure in these metals, one need only deposit a film of sufficient time to confidently 

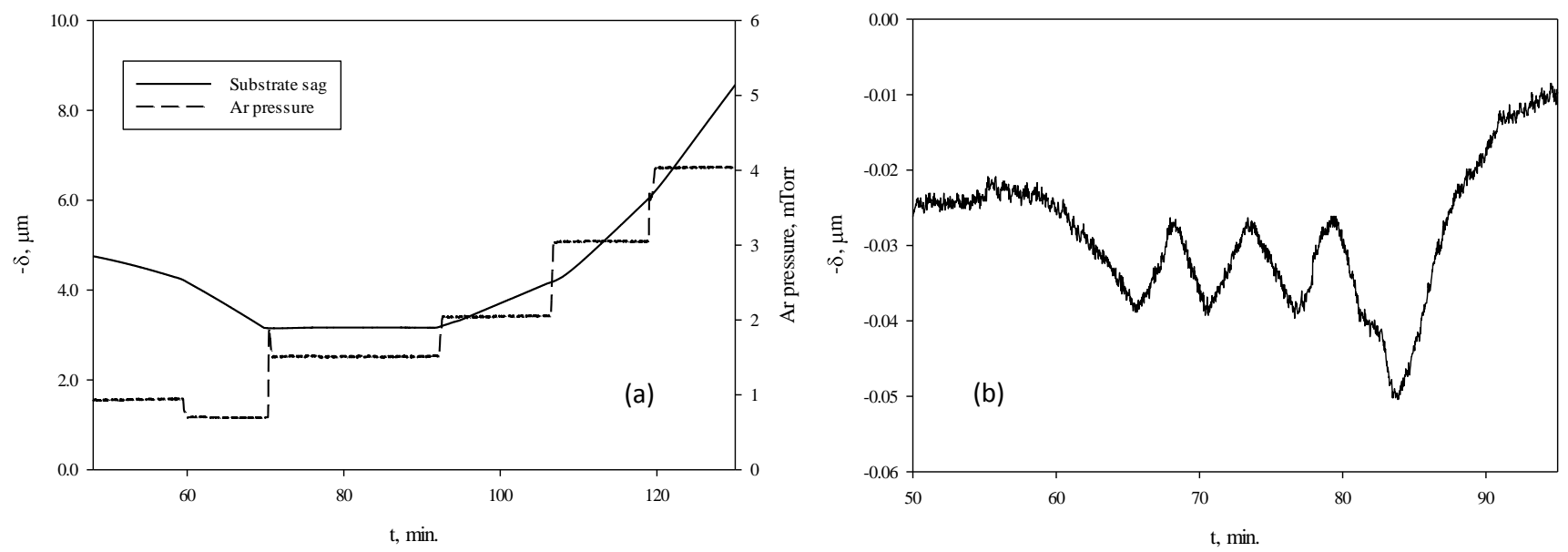

Figure 3. (a) Plot illustrating the tuning of the argon process pressure for achieving near-zero instantaneous in $\mathrm{Cr}$. (b) The modulation of the instantaneous stress about the $\mathrm{Cr}$ critical pressure of $\sim 1.60 \mathrm{mTorr}$. The $\pm 0.02 \mathrm{mTorr}$ changes in the process pressure resulted in a corresponding change of the instantaneous stress of $\mp 9.36 \mathrm{MPa}$.

determine the ensuing constant slope, $\mathrm{d} \delta / \mathrm{dt}$. In this way, the general dependence of the intrinsic stress with argon pressure, for example, can be quickly realized in a single deposition run, or the stress "tuned" to near-zero.

Figure 3(a) illustrates the capability of maintaining near-zero stress in $\mathrm{Cr}$ with tuning of the argon pressure. The instantaneous stress was maintained at an average value of $\sim 2.4 \mathrm{MPa}$ for a twenty minute interval. The critical value of argon pressure for achieving near-zero stress in $\mathrm{Cr}$ is $\sim 1.60 \mathrm{mTorr}$. The method of stress tuning has been envisioned as at least one possible approach for achieving low values of stress that is needed during the differential deposition process. For example, the stress could first be tuned to zero by first depositing on a sensor located in a similar geometric orientation near the surface of the substrate to be corrected.

In the differential deposition process, up to several hundred nanometers are deposited to correct mid-spatial-frequency figure errors of the thin full-shell replicated substrates which can result from the relaxation of electroforming stresses or from figure errors present in the formed mandrel ${ }^{16}$. This deposition technique, however, relies in part on a nearly stressfree film. Otherwise, the stress in the figure-correcting layer can itself cause stress-induced distortions of the mirror. For full-shell nickel replication, the sputtered figure correcting material is currently Ni since the extrinsic stress due to the coefficient of thermal expansion (CTE) difference between the film and substrate is expected to contribute negligibly to the total stress. However, this material has not yet been fully vetted in regard to its surface roughness and long term environmental and thermal stability. A more detailed evaluation of this material is forthcoming.

The capability to maintain a near-zero value of stress using gas pressure is limited by the resolution of its control and is process system specific. The sensitivity in the incremental stress to changes in process pressure around the transition point is high (i.e. $936 \mathrm{MPa} / \mathrm{mT}$ Torr); illustrating the benefit of in-situ monitoring of the film stress. Figure 3(b) illustrates the modulation of the instantaneous stress due to the forced oscillatory changes in the argon pressure of \pm 0.02 mTorr about the critical pressure. The high-frequency random variation of the displacement that is associated with the $\pm 5 \mathrm{~nm}$ resolution limit of the sensor is evident in the figure since the total displacement is a few tens of nanometers. The transition between the constant compressive and tensile incremental stress corresponded to values of argon process pressure of 1.61 and $1.63 \mathrm{mTorr}$ which resulted in incremental stress values of $\mp 9.36 \mathrm{MPa}$, respectively. An improvement in the resolution in controlling the gas pressure (i.e. better than $\pm 0.02 \mathrm{mTorr}$ ) would be needed for a proportional reduction of the variation band of the instantaneous stress. The results of figure 3(b) clearly demonstrate that the sensitivity of the in-situ instrument far exceeds the resolution in the control of the argon process pressure. 


\subsection{Instantaneous stress in $\mathrm{Cr}$, $\mathrm{Ni}$, and Ir thin films}

Shown in Figure 4(a) is a comparison of the intrinsic instantaneous stress in $\mathrm{Cr}, \mathrm{Ni}$, and $\mathrm{Ir}$ with gas pressure. The coatings were deposited at a similar deposition rate of $10-13 \mathrm{~nm} / \mathrm{min}$. The pressure which results in zero stress (i.e. critical pressure) scales with the density of the material. Among these three materials, $\mathrm{Cr}$ has the lowest density and hence the lowest critical pressure. The low value of critical pressure for $\mathrm{Cr}$ is likely why tensile stress is often associated with this material. In fact, the stress in $\mathrm{Cr}$ can be forced to the compressive regime for gas pressures below $\sim 1.5 \mathrm{mTorr}$. The stress reversal in $\mathrm{Cr}$ has been reported previously ${ }^{6}$ and the results qualitatively consistent with our measurements, including the tensile maxima that occurs in the 4-5 mTorr range. It is evident then, that a new alternative for achieving a balance in the iridium stress for slumped glass segments might be achieved by depositing the $\mathrm{Cr}$ and $\mathrm{Ir}$ layers on opposing surfaces. That is, the iridium layer is deposited on the concave surface, while the compressive $\mathrm{Cr}$ (or some other compressively stressed material) layer is deposited on the opposing, convex surface. In this way, the problem of increased surface roughness which would otherwise result from the $\mathrm{Cr} / \mathrm{Ir}$ bi-layer approach is mitigated. We have successfully deposited $\mathrm{Cr}$ in excess of a several hundred nanometers without delamination due its excellent adhesion.
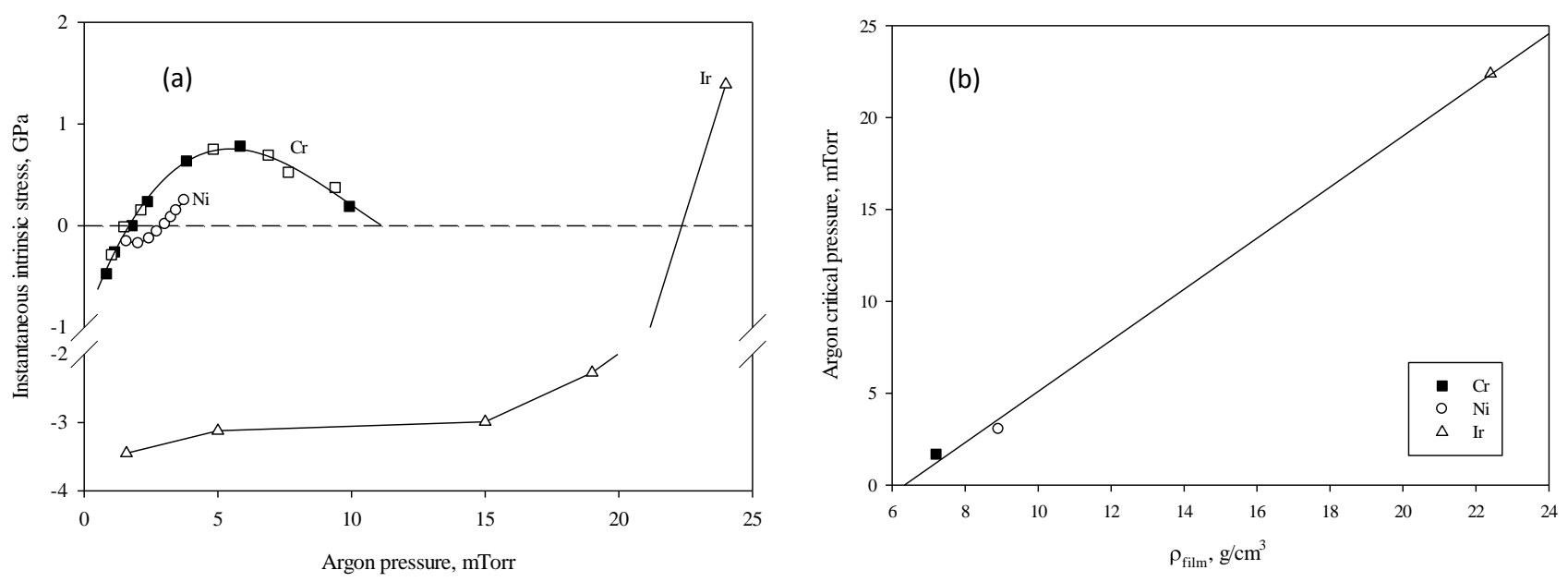

Figure 4. (a) Comparison of the instantaneous stress in $\mathrm{Ni}, \mathrm{Cr}$, and Ir film with argon process pressure. (b) Scaling of the critical pressure stress with the density of the sputtered material. The critical pressure of $\mathrm{Cr}$ is $\sim 1.6$ mTorr. Pressures below this value will result in intrinsic compressive stress.

The large value of critical pressure (i.e. 20-22 mTorr) resulting from iridium's high density could make its discovery difficult a posteriori. The characteristic features of the in-situ stress for metal films has been correlated with different growth modes (i.e. island or columnar) due to the influence of the deposition parameters on the adatom mobility? Pressures above the critical pressure result in low adatom mobility due to energy losses as a consequence of gas scattering of the transiting sputtered atom from target to substrate. This energy loss results in a decrease in the adatom mobility and the microstructure of the film is columnar. For $\mathrm{Cr}$, this regime of film growth occurs at low process pressures and the rate of an increase in roughness with gas pressure and film thickness is large. Care should be taken not to equate a similar outcome to high density materials such as Ir where a much larger separation between these growth regimes exists. In the sections to follow we will examine the surface roughness and stress in Ir films in the transitional region between, what Abermann referred to as Type I (low adatom mobility) and Type II (high adatom mobility) growth regimes. 


\subsection{The total in-situ internal stress}

The determination of stress during film growth is clearly advantageous since the inclusive resultant internal stress can be resolved into its intrinsic and extrinsic components as shown in Eqn. (6). The former of the two

$$
\sigma_{t o t}=\sigma_{i}+\sigma_{\triangle C T E}
$$

is of much interest since it reveals information about the microstructural composition of the film, which can often be associated with a relevant application-specific performance characteristic(s) — such as surface roughness. The extrinsic component is typically associated with the temperature-related strain brought about by the mismatch in CTE between the film and substrate. This quantity necessarily arises as a consequence of the heat generated during deposition or annealing processes and also depends on the heat transfer characteristics of the film and substrate. For the special case in which the substrate is actively maintained at a constant temperature throughout the deposition process, the extrinsic stress in the film after the substrate cools to room temperature is given by,

$$
\sigma_{\triangle C T E}=M_{f}\left(\alpha_{s}-\alpha_{f}\right) \Delta T
$$

Here $\mathrm{M}_{\mathrm{f}}$ is the film's biaxial modulus and $\alpha_{\mathrm{s}}$ and $\alpha_{\mathrm{f}}$ are the respective CTE of the substrate and film, while $\Delta \mathrm{T}$ is the difference between the deposition and room temperatures.

Otherwise, the calculation of the thermal component of curvature change must account for temperature change both during and after deposition. The portion of the total curvature change during the deposition process due to thermal stress must be subtracted in order to obtain the intrinsic component. We are working on a method for extracting the intrinsic component of stress for a substrate whose temperature is change during the deposition process. This method is based on in-situ measurement of the substrate temperature during the deposition process. We are in the process of developing potential improvements to this method, including the sensitive non-contact acquisition of the substrate temperature, which will be presented at a later time. For now, however, it should be understood that the thermal contribution to the total stress is also contained in we have denoted as the intrinsic stress, $\sigma_{\mathrm{i}}$. For most cases the thermal stress is a higher order contributing effect in comparison to the dominant intrinsic behavior associated with the characteristic features of early film growth - particularly for iridium. The thermal component of the contribution to the total substrate curvature during deposition is more prominently revealed for other materials, such as $\mathrm{Ni}$, that has a large thermal expansion coefficient and inherently low intrinsic stress.

\subsection{Correction of the measured displacement due to systematic error}

The instantaneous stress shown in Fig (4) was obtained for a thermally equilibrated substrate. The time required for the substrate to reach thermal equilibrium results in a film thicknesses of several hundred nanometers greater than needed for $\mathrm{x}$-ray optical coatings. To measure the stress in films which are only tens of nanometers thick, the displacement measurement must be corrected to account for a systematic temperature-related error. These errors arise as a consequence of the thermal expansion of the substrate support mechanism and optical probe tip under the heat load of the plasma. To subtract this error a thermocouple is positioned in one of the support legs upon which the substrate rests to provide in-situ measurement of a temperature related to the substrate. This method of substrate temperature measurement does not inadvertently influence the substrate's curvature. This temperature, denoted as $\mathrm{T}_{\mathrm{r}}$, is well correlated to the linear displacement error which has been found through active radiative heating of an uncoated substrate. While this temperature is related to the substrate temperature, there is some offset which is $\sim 33 \%$ below the true substrate temperature. Nonetheless, the relative change in this temperature scales linearly and reversibly with the displacement error (i.e. $0.015 \mu \mathrm{m} /{ }^{\circ} \mathrm{C}$ ). 

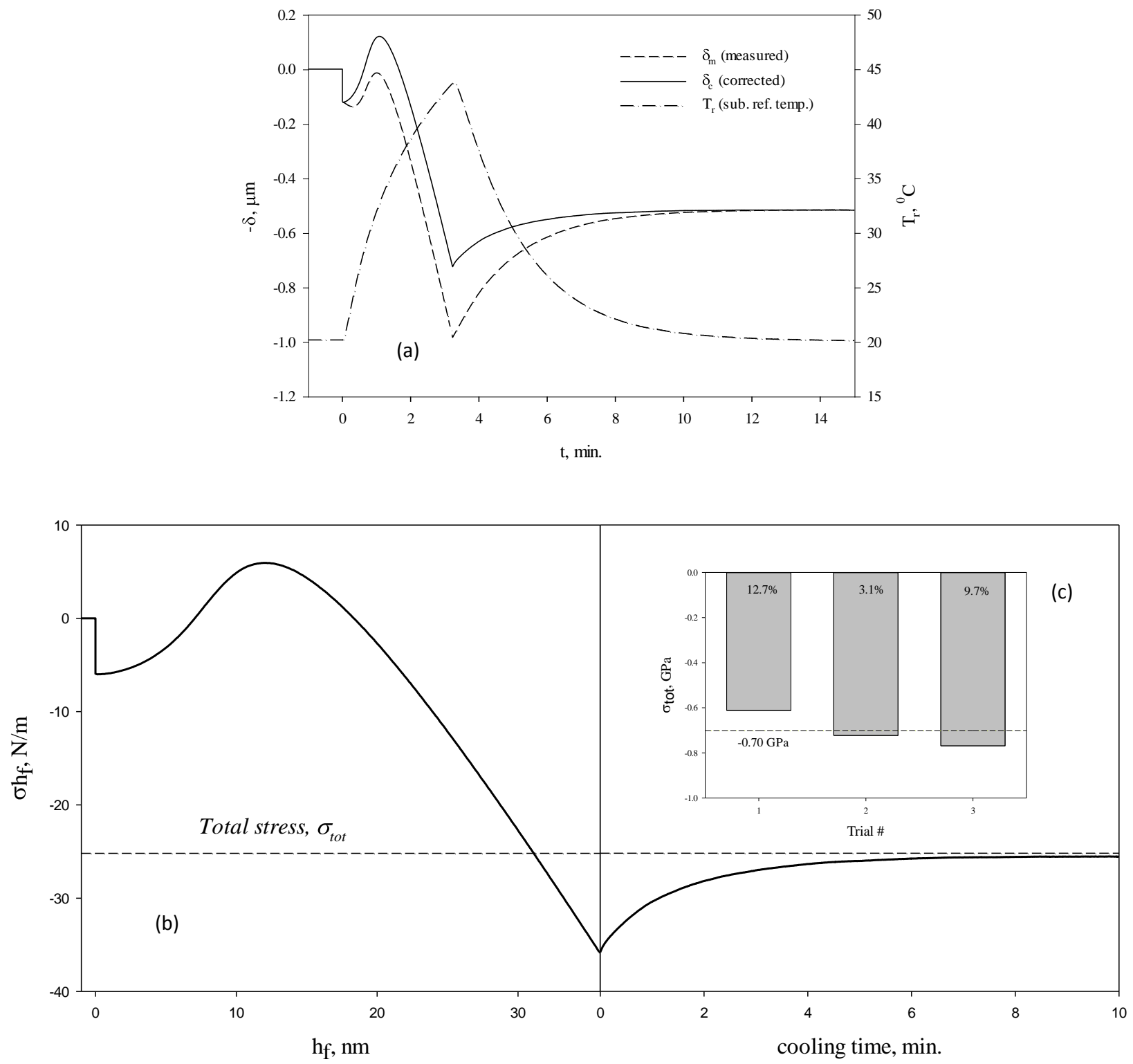

Figure 5. (a) Comparison of the raw, corrected, and substrate reference temperature for an iridium film deposited at $19.0 \mathrm{mTorr}$ at a deposition rate of $13 \mathrm{~nm} / \mathrm{min}$. A linear systematic error of $0.015 \mu \mathrm{m} /{ }^{\circ} \mathrm{C}$ results as a consequence of the thermal expansion of the substrate support mechanism. (b) The force per unit width resulting after correction of the displacement measurement. The $0.1{ }^{\circ} \mathrm{C}$ resolution in the measurement of the reference temperature limits the minimum detectable force per unit width to $0.035 \mathrm{~N} / \mathrm{m}$. (c) The percent deviations from the average total stress of $-0.70 \mathrm{GPa}$ for three repeated deposition runs at $19.0 \mathrm{mTorr}$.

Comparison of the raw, corrected, and reference temperature data for an iridium film deposited at $13.0 \mathrm{~nm} / \mathrm{min}$ at an argon pressure of 19.0 mTorr is shown in Fig. 5(a). The linear dependence of the measurement error, $\delta_{\varepsilon}\left(T_{r}\right)$, is subtracted from the measured displacement via in-situ measurement of the reference temperature. The raw and uncorrected data is in agreement when the substrate cools back to room temperature. This ending displacement corresponds to a value that would be measured using ex-situ methods, for example. A future improvement to the device 
will include a second displacement sensor located on the substrate support periphery which will provide a direct measurement of the systematic error.

Figure 5(b) shows the resulting force per unit width after application of Eqn. (4) to the corrected displacement data. A notable feature in the data is the existence of a discontinuity in the displacement the moment deposition first begins, (i.e. $\mathrm{t}=0$ ). We have found the magnitude of this discontinuity to be proportional to the deposition rate and inversely proportional to the substrate thickness. Initial calculations show that these observations are likely consistent with thermal shock of the substrate. Further, the magnitude of the displacement is also predicted to scale with substrate area which might explain why this observation has not been noted in other published work where micro-cantilevers are utilized.

\subsection{Measurement repeatability}

Figure 5(c) shows the deviation in the total stress from the average of three repeated deposition runs. These values represent not only the repeatability of the measurement of the substrate's displacement, but also the repeatability of the deposition process parameters. The stress depends on the repeatability of several deposition parameters including: substrate temperature, deposition rate, argon pressure, and substrate bias, for example. It is thought that the run-to-run variation of the deposition process dominates the variation in the measurement of the substrate's displacement. While we realize what is shown in Fig. 5(c) does not constitute a rigorous statistical analysis of the measurement repeatability of our devise, it is nonetheless reasonable to expect that the repeatability of our instrument should be substantially better than ex-situ measurement techniques that utilize profilometry, for example. The total stress that would be measured by ex-situ methods is indicated in Fig. 5(b) with the dashed line. The largest source of error in ex-situ measurement methods is associated with tracing, removing, depositing, indexing and re-retracing the same location on the substrate. The large variation in the measurement of the stress associated with ex-situ measurement can often be correlated to the variation in the initial curvature of the substrates for a given indexing repeatability between traces. Ex-situ techniques can be particularly unforgiving when attempting to measure near-zero value of film stress. These difficulties are largely overcome when in-situ measurement is utilized.

\subsection{The in-situ intrinsic stress}

During early growth of the film in the high adatom (Type II) mobility regime, the stress is initially compressive, reaches a tensile maximum, passes through zero, then returns to long term compression. The tensile maximum for the case of high adatom mobility is associated with the completion of island coalescence. These growth features are absent for the low adatom mobility (Type I) regime in which case the force per unit width increases monotonically. Columnar structure of the film is associated with this regime of film growth were high surface roughness is expected to ensue. In each case a linear displacement of the force per unit width with film thickness eventually remains--indicating that the stress in the growing film thereafter remains constant.

\subsubsection{Approaches for achieving zero stress in iridium}

The intrinsic stress behavior of the films versus argon pressure is shown in Fig. 6(a). Note the broadening of the tensile maximum that occurs with increasing argon pressure. It is suspected that for an optimal gas pressure the dependence of the intrinsic stress with film thickness would eventually flatten to zero. This critical process pressure marks the boundary of the entrance into the low adatom mobility growth regime; where afterward, the surface roughness is expected to increase substantially.

A second approach for achieving zero stress at comparatively lower process pressure is to take advantage of the film stress' passage through zero after island coalescence and prior to reaching a compressive steady state. The value of film thickness at which this occurs can be controlled with argon pressure, as observed in Fig. 6(a). In this way, one can 
achieve zero stress at lower gas pressure which might be favorable for reducing the film's surface roughness. Figure 6(b) shows that the critical pressure for achieving zero intrinsic stress in a $10 \mathrm{~nm}$ thick film is about $13.0 \mathrm{mTorr}$, for example. The film's thickness will have to be increased somewhat beyond $10 \mathrm{~nm}$ to account for the tensile component of the thermal strain upon cooling. This is counterintuitive since an increase in the film's thickness is needed in order to reduce its total internal stress.
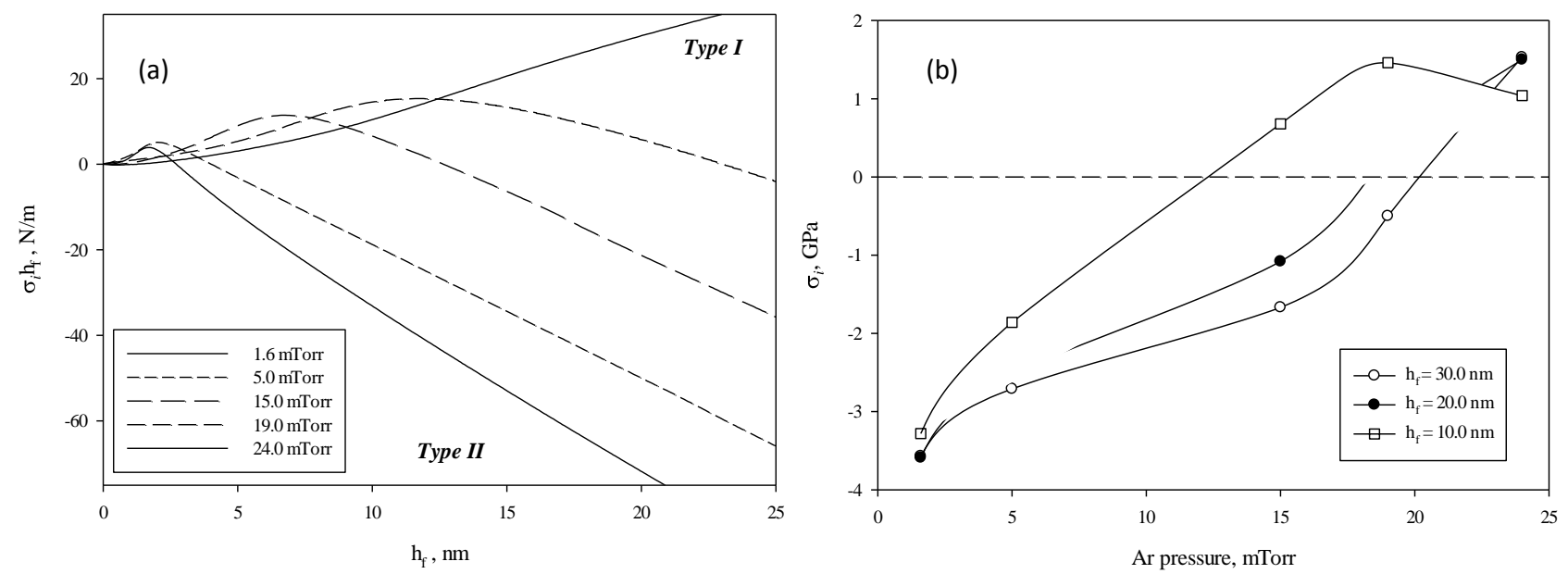

Figure 6. (a) The intrinsic force per unit width for iridium deposited at various argon gas pressures. The figure illustrates the behavior during film growth for high (Type II) and low (Type I) adatom mobility. The stress passes through zero shortly after the completion of island coalescence (b) The intrinsic stress for film thicknesses of 30, 20, and $10 \mathrm{~nm}$ at the indicated gas pressure is shown.

\subsection{Zero stress in the steady-state regime of film growth}

Clearly, we have demonstrated the ability to achieve near-zero values of the internal stress in iridium films through the optimization of the argon process pressure. Achieving near-zero values of film stress in iridium, however, provides only a partial solution. The complete solution requires near-zero stress and acceptable surface roughness appropriate to soft $\mathrm{x}$-ray wavelengths.

In order to evaluate the feasibility of using our approach for reducing the stress in iridium, several samples were deposited on silicon substrates and measured using XRR (x-ray reflectometry) at SAO. The reflectivity was modeled using IMD to extract the film thickness, surface roughness, and film density. The value of surface roughness resulting from this analysis is shown in Fig. 7(a) along with the corresponding value of total stress achieved at the indicated process pressures. The deposition rate and maximum substrate temperature were also affected by changes in the argon pressure as indicated in Fig. 7(b). These quantities are also expected to have a degree of impact on the values of internal stress, although smaller in comparison to the influence of argon pressure. The films ranged in thick from 31.2-45.5 nm. Referring to Fig. 7(a), the critical pressure can be observed to occur at about 22 mTorr in steady-state stress regime. Argon pressures above this value result in tensile stress and a significant increase in surface roughness. Surprisingly, however, the surface roughness is reasonably well behaved and a modest rise between $4-6 \AA$ is observed to occur over a broad range of argon pressure in the 10-20 mTorr range.

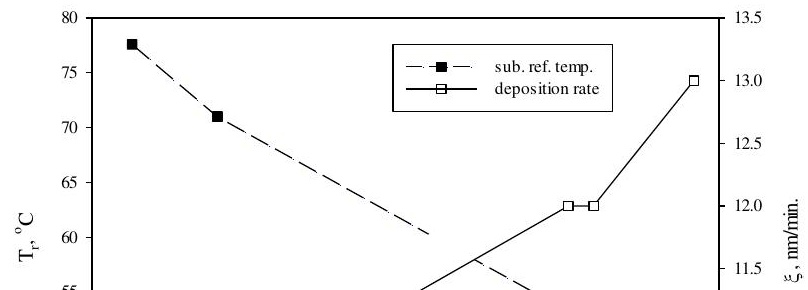




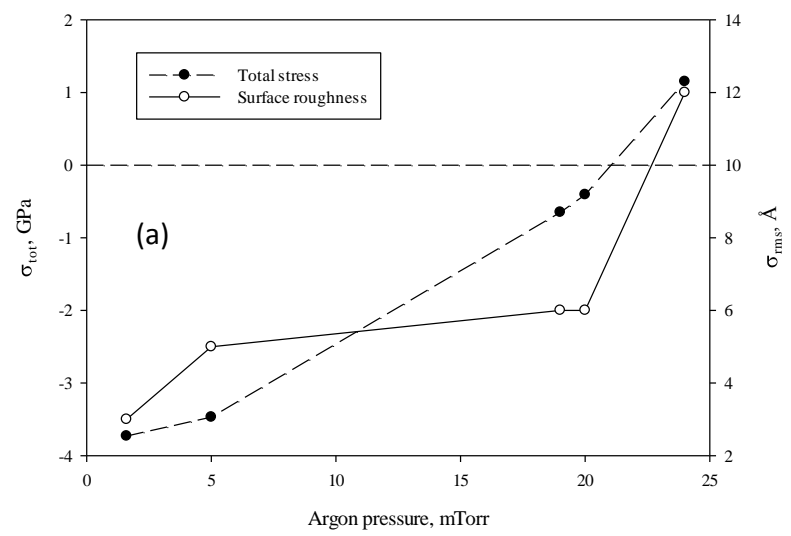

(b)

Figure 7. (a) Plot comparing the total internal stress and the film's surface roughness. The surface roughness was evaluated from XRR reflectivity at $\mathrm{CuK} \alpha$. (b) The dependence of deposition rate and substrate temperature versus argon pressure is shown.

\subsubsection{Zero stress shortly after island coalescence}

A film thickness of about $10.0 \mathrm{~nm}$ is adequate for achieving good $\mathrm{x}$-ray reflectivity. For lower film thickness then, one can take advantage of the growth features of the film in order to achieve zero stress at lower argon pressure. To this aim, we deposited a $15.8 \mathrm{~nm}$ iridium film at $15.0 \mathrm{mTorr}$. The film was deposited past the zero intrinsic stress value to compensate for the extrinsic thermally driven tensile component. The in-situ stress for the process parameters is shown in Fig. 8 where we have measured a total internal stress of -2.89 MPa. XRR measurement and analysis of this sample at SOA revealed an RMS surface roughness of $\sim 5.0 \pm 0.5 \AA$ and film density close to the bulk. The adhesion of the film survived a standard tape pull test.

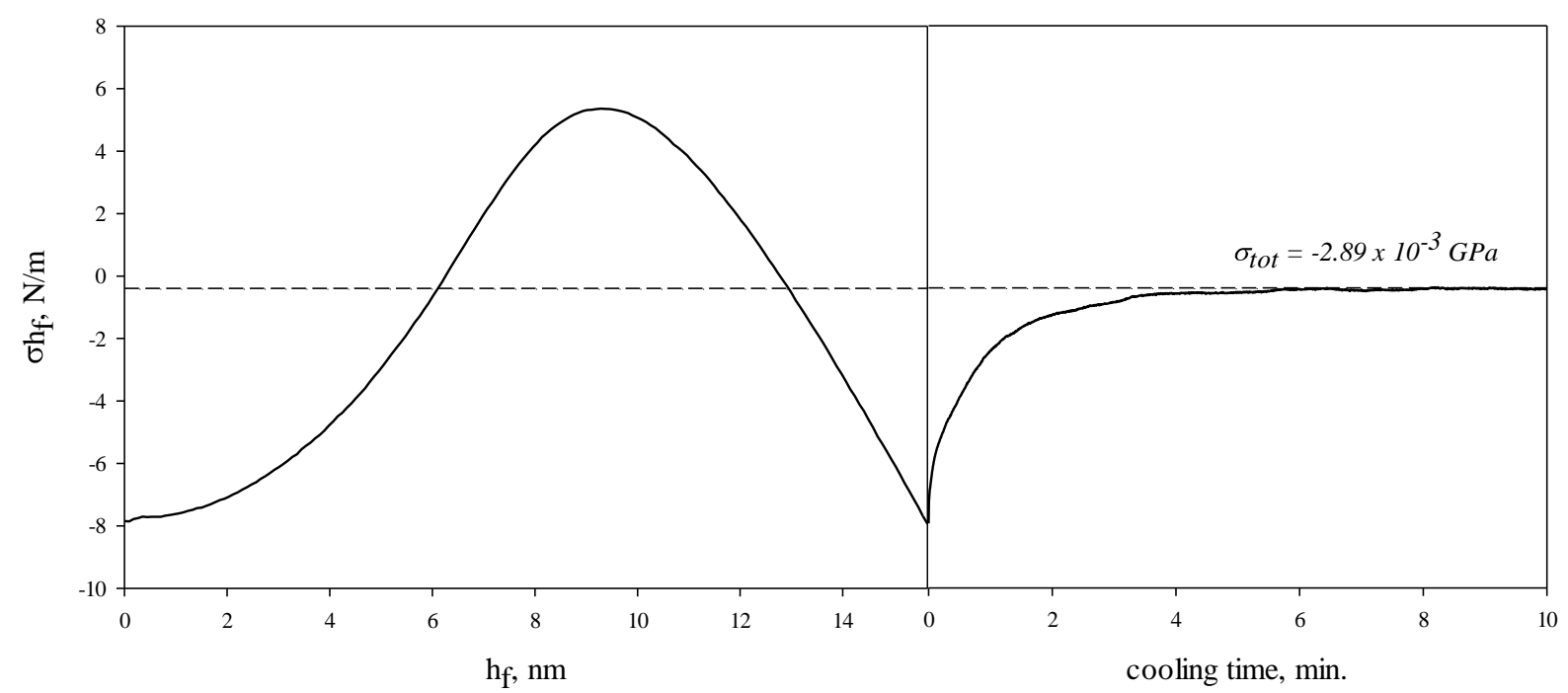

Figure 8. The in-situ film stress of iridium deposited at $15.0 \mathrm{mTorr}$ at a deposition rate of $\sim 11 \mathrm{~nm} / \mathrm{min}$. The total internal stress was $-2.89 \mathrm{MPa}$, which is a reduction in nearly three orders of magnitude from the stress deposited at the lowest argon pressure of 1.6 mTorr.

\section{Surface roughness evaluation of low-stress iridium}




\subsection{Scanning probe microscopy (SPM)}

Scanning probe images were obtained with a Dimension (Bruker) D3000 system in tapping mode using nanoprobe tips operating at $260-275 \mathrm{kHz}$ frequency. The $\mathrm{x}, \mathrm{y}$, and $\mathrm{z}$ distances were calibrated to a nominal $\pm 2 \%$ relative accuracy. Scans were taken at $10 \mathrm{um}$ x $10 \mathrm{um}(0.7 \mathrm{~Hz}), 1 \mathrm{um}$ x $1 \mathrm{um}(1.5 \mathrm{~Hz})$, and $0.1 \mathrm{um}$ x $0.1 \mathrm{um}(2.3 \mathrm{~Hz})$ on three different regions for each coating and one region on the glass. Resolution was 512 x 512 points in $\mathrm{x}, \mathrm{y}$ and the z-control response (resolution) was increased by a factor of 7.7 over default. All images were pre-processed by a second-order flatten operation before roughness values and power spectral density (PSD) curves were measured using version 6.13r1 of the D3000 software. 
[1] Ferreira, D.M., Christensen, F.E., Jakobsen, A.C., Westergaard, N.J., “ATHENA optimized coating design", Proc. of SPIE 8443 (2012)

[2] Ferreira, D.M, Jakobsen, A.C., Christensen, F.E., Shortt, B., Krumrey, M., Garnaes, J., Simonsen, R.B., "Development and characterization of coatings on Silicon Pore Optics substrates for the ATHENA mission", Proc. SPIE 8443 (2012)

[3] Chan, K.W., Sharpe, M., Zhang, W., Kolos, L., Hong, M., McClelland, R., Hohl, B.R., Saha, T., Mazzarella, J., "Coating thin mirror segments for lightweight X-ray optics”, Proc. SPIE 8861 (2013).

[4] Paul B. Reid, Thomas L. Aldcroft, Vincenzo Cotroneo, William Davis, Reagan L. Johnson-Wilke, Stuart McMuldroch, Brian D. Ramsey, Daniel A. Schwartz, Susan Trolier-McKinstry, Alexey Vikhlinin, and Rudeger H. T. Wilke, "Technology development of adjustable grazing incidence X-ray optics for sub-arc second imaging", Proc. SPIE 8443, (2012).

[5] Paul B. Reid, Thomas L. Aldcroft, Vincenzo Cotroneo, William Davis, Reagan L. Johnson-Wilke, Stuart McMuldroch, Brian D. Ramsey, Daniel A. Schwartz, Susan Trolier-McKinstry, Alexey Vikhlinin, and Rudeger H. T. Wilke, "Development status of adjustable grazing incidence optics for 0.5 arc second X-ray imaging”, Proc. SPIE Vol. 8861 (2013)

[6] D.W. Hoffman, “Internal stresses of sputtered chromium”, Thin Solid Films, 40 (1977) 355-363.

[7] Thorton, J.A., Hoffman, D.W., "Stress-related effects in thin films”, Thin Solid Films, 171 (1989) 5-31

[8] John A. Thornton, David W. Hoffman, "Internal stresses in titanium, nickel, molybdenum, and tantalum films deposited by cylindrical magnetron sputtering”, J. Vac. Sci. Tech., Vol. 14, No.1, Jan/Feb. 1977

[9] Abermann, R., "Measurements of the Intrinsic Stress in Thin Metal Films", Vacuum, Vol. 41, 4-6, pg. 1279-1282, (1990)

[10] Broadway, D.M, "Mechanical stress measurement during thin film fabrication", NASA, United States Patent Application \#14,645,994, (2015)

[11] Janssen, G.C.A.M., Abdalla, M.M., van Keulen, F., Pujada, B.R., van Venrooy, B., "Celebrating the $100^{\text {th }}$ anniversary of the Stoney equation for film stress: Developments from polycrystalline steel strips to single crystal silicon wafer”, Thin Solid Films, 517, (2009), 1858-1867.

[11] Floro, J.A., Chason, E., Lee, S.R., Real Time Measurement of Epilayer Strain Using a Simplified Wafer Curvature Technique, Mat. Res. Soc. Symp. Proc. Vol. 406 (1996)

[12] Floro, J.A., Chason, E., Lee, S.R., Twesten, R.D., Hwang, R.Q., Real-Time Stress Evolution During Si1xGex Heteroepitaxy: Dislocations, Islanding, and Segregation, Journal of Electronic Materials, Vol. 26, No.9, (1997) 
[13] Abermann, R., "Measurements of the Intrinsic Stress in Thin Metal Films", Vacuum, Vol. 41, 4-6, pg. 1279-1282, (1990)

[14] Klokholm, E., Berry, B.S., Internal Stress in Evaporated Metal Films, J. Electrochem. Soc., 115 (1968) 823-826.

[15] Klokholm, E., An Apparatus for Measuring Stress in Thin Films, Review of Scientific Instruments, (1969) Vol. 40, No.8, 1054-1058

[16] Kilaru, K., Ramsey, B.D., Gubarev, M.V., \& Gregory, D.A., Differential deposition technique for figure corrections in grazing-incidence X-ray optics, OptEn 50, $1065016 \mathrm{pp}$ (2011). 